\title{
Project 8: R\&D for a next-generation neutrino mass experiment
}

\section{René Reimann* for the Project $\mathbf{8}$ collaboration}

Institute of Physics \& PRISMA ${ }^{+}$Cluster of Excellence, Johannes Gutenberg-University Mainz, Staudinger Weg 7, 55099 Mainz, Germany

E-mail: rreimann@uni-mainz.de

Project 8 is a next-generation direct neutrino mass experiment measuring the spectral endpoint region of tritium beta decays. The energy of the beta decay electrons is measured using Cyclotron Radiation Emission Spectroscopy (CRES) which has been demonstrated by the Project 8 collaboration with both krypton and molecular tritium confined in a section of a microwave guide. To reach the target sensitivity of $40 \mathrm{meV} / \mathrm{c}^{2}$, major technological research and development is necessary. Building on the milestones achieved so far, I will present the next phases of Project 8: In Phase III, atomic tritium will be produced and trapped magnetically, and cyclotron radiation emission spectroscopy will be demonstrated in free space which allows larger detection volumes. The knowledge gained from Phase III will enable the design and operation of a large-volume atomic tritium experiment, sensitive to the entire mass range allowed by the inverse neutrino mass ordering.

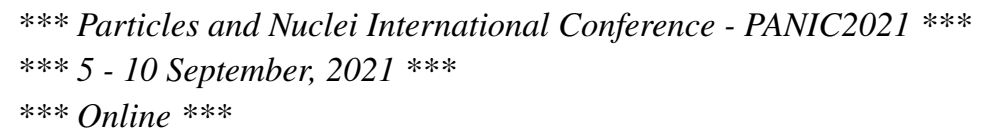

${ }^{*}$ Speaker 


\section{Challenges in Direct Neutrino Mass Experiments}

Neutrino flavour oscillation has established that at least two neutrino eigenstates have nonzero masses. However, oscillation experiments can only determine the differences of neutrino masses squared and thus the absolute scale of neutrino masses is unknown. Experimentally the absolute neutrino masses can be determined using cosmology models and data, as neutrinos alter the evolution of structure formation, using neutrinoless double beta decays, in case the neutrino is its own anti-particle or by precisely measuring the end-point region of a beta or electron capture spectrum.

While the neutrino mass measurements using cosmological models and neutrinoless double beta depend on the specific model that was used to derive the neutrino masses, the signature of neutrino mass at the spectrum's endpoint is based on a purely kinematic effect and thus does not depend on any model assumption for simple nuclei. The endpoint energy is defined by the difference in binding energies of the parent and daughter nucleus. In case of zero neutrino mass, the decay electron spectrum ranges up to the endpoint energy. However if the neutrino mass is non-zero the spectrum endpoint is shifted and the shape and amplitude close to the spectrum endpoint is altered [1]. This was already pointed out by Fermi in his original paper about the theory of $\beta$ decays [2]. The spectrum endpoint method is sensitive to the flavor-weighted sum of neutrino masses

$$
m_{\beta}=\sqrt{\sum_{i=1}^{3}\left|U_{e i}\right|^{2} m_{i}^{2}},
$$

where $U_{e i}$ are the coefficients from the Pontecorvo-Maki-Nakagawa-Sakata matrix and $m_{i}$ are the neutrino masses. As differences of neutrino mass squared are known from oscillation experiments, $m_{\beta}$ has a minimal possible value. In case of inverted ordering $m_{\beta}$ cannot be lower then $\sim 40 \mathrm{meV} / \mathrm{c}^{2}$.

Most direct neutrino mass experiments use the decay spectrum of tritium ${ }_{1}^{3} \mathrm{H} \rightarrow{ }_{2}^{3} \mathrm{He}^{+}+e^{-}+\bar{v}_{e}$ which has a relatively low endpoint energy of $\sim 18.6 \mathrm{keV}$. At lower endpoint energy it gets simpler to achieve the required energy resolution, and the neutrino mass effect is a larger fraction of the total spectrum. The tritium is gaseous and its decay is a super-allowed decay with a half-life of $12.3 \mathrm{yr}$.

The current leading limits on neutrino mass from direct mass measurements are from the KATRIN experiment, which uses the magnetic adiabatic collimation combined with an electrostatic filter (MAC-E filter) technique and the first sub eV limits have been shown recently [3]. KATRIN's final sensitivity goal is $\sim 200 \mathrm{meV}$ which is well above the minimal possible value of $m_{\beta}$. In direct mass measurements the statistical sensitivity scales with $N^{-1 / 4}$, where $N$ is the number of detected electrons from beta decay in the region of interest, and the existing detector technology reached its limit of scalability. In addition, irreducible systematic effects like spectrum broadening due to molecular final states caused by rotational and vibrational excitation in $\mathrm{T}_{2}$ molecules limit the sensitivity to $\sim 100 \mathrm{meV}$.

\section{The Project 8 Experiment}

The Project 8 experiment is a next-generation direct neutrino mass measurement also based on the end-point technique for beta decays, that uses a new technology called cyclotron radiation 
emission spectroscopy (CRES) proposed by [4]. This technique uses the frequency-energy relation for relativistic electrons

$$
f_{c}=\frac{f_{c, 0}}{\gamma}=\frac{1}{2 \pi} \frac{e B}{m_{e}+E_{\mathrm{kin}} / c^{2}},
$$

where $f_{\mathrm{c}}$ is the cyclotron frequency, $\gamma$ is the Lorentz factor, $B$ is the magnetic flux density, $E_{\text {kin }}$ is the kinetic energy of the decay electron and $e$ and $m_{e}$ are the charge and mass or the electron, respectively. For a kinetic energy of a decay electron close to the tritium endpoint of $\sim 18.6 \mathrm{keV}$ and a magnetic field of $\sim 1 \mathrm{~T}$, the electron radiates $\sim 1 \mathrm{fW}$ at the cyclotron frequency of $25.9 \mathrm{GHz}$ which is in the microwave regime. While the small amount of radiated power makes it challenging to detect electrons, the method has several advantages: 1. This method turns the energy into a frequency measurement, which allows for a high precision measurement. 2. As the tritium gas is transparent to its own microwave radiation, the source volume is also the detection volume and decay electrons do not have to be transported. This allows the volume to be scaled up. 3 . This technique gives a differential spectrum which increases the statistical efficiency compared to integrating spectrometers. 4. This technique is compatible with atomic tritium, which avoids final-state spectral broadening of $\mathrm{T}_{2}$.

To establish a neutrino mass experiment with a final sensitivity of $\sim 40 \mathrm{meV} / \mathrm{c}^{2}$ using the CRES technique, the Project 8 collaboration follows a phased approach.

In Phase I, the CRES technique was demonstrated and the first single CRES signals were detected. The apparatus consists of a waveguide which is also decay and detection volume and read out by a cryogenic amplifier cooled by a mechanical cryocooler. A superconducting solenoid magnet produced a homogeneous background field. In addition pinch coils were used to build a magnetic trap for the decay electrons which increases the observation time. A single CRES signal can be seen as a trace of excess RF power bins in a spectrogram [5]. The start frequency in the spectrogram gives the birth kinetic energy of the electron and the cyclotron radiation loss is visible as a slow chirp in frequency. Collisions with residual gas lead to abrupt energy losses and changes in direction which introduce discrete jumps in the spectrogram. By measuring the conversion-electron spectrum from ${ }^{83 m} \mathrm{Kr}$ it was shown that the CRES technique can be used for high-resolution spectroscopy [6].

In Phase II the apparatus was similar to Phase I with an effective volume of $1 \mathrm{~mm}^{3}$. Measurements with ${ }^{83 m} \mathrm{Kr}$ and $\mathrm{T}_{2}$ allowed studies of systematics and backgrounds [7]. The first $\mathrm{T}_{2}$ spectrum and endpoint measurement analyzed using a Frequentist and Bayesian analysis, including instrumental resolution, scattering effects, detection efficiency as function of frequency and ${ }^{83 m} \mathrm{Kr}$ calibration have been recently shown [8].

Phase III is devoted to developing all remaining technologies that are needed for a full scale next generation neutrino experiment. Current efforts are discussed in the next section.

Phase IV will be the full scale neutrino mass experiment with a $40 \mathrm{meV} / c^{2}$ neutrino mass sensitivity. This allows the determination of absolute neutrino masses in case of inverted ordering or rules out inverted ordering as neutrino mass hierarchy. 


\section{R\&D efforts for Phase III}

Having demonstrated a neutrino mass measurement with molecular tritium in the limited volume of a waveguide with about $\sim 1 \mathrm{~mm}^{3}$ effective volume, two major challenges can be identified on the path to a next-generation neutrino mass experiment. First, the effective volume has to be scaled up to increase the statistics. Second, the technique has to be demonstrated to work with atomic tritium which requires an atomic tritium source. In a first step both challenges are addressed separately by two technology demonstrator setups.

\subsection{Free Space CRES Demonstrator}

The aim of the Free Space CRES Demonstrator (FSCD) is to leave the restricted volume of a waveguide with $1 \mathrm{~mm}^{3}$ effective volume and demonstrate the CRES technique in free space with effective volumes of $10-100 \mathrm{~cm}^{3}$. To achieve this, $R \& D$ work is ongoing:

Antenna design: In the FSCD the radiated power of the electron is detected using antennas working in the far field regime. For this effort patched antenna arrays and slotted waveguides have been modeled and comparison of simulation with prototype measurements are performed. Alternatively, resonant cavity designs are being explored.

Low noise amplifiers: To achieve sufficient signal/noise ratios given the tiny radiated signal power, low noise amplifiers are needed. Josephson Traveling Wave Parametric Amplifier working near quantum-noise limit show high gain over broad frequency range ( $20 \mathrm{~dB}$ over $2 \mathrm{GHz}$ ) [9]. However their performance at $26 \mathrm{GHz}$ is unknown and multiplexing is not validated at frequencies above $>10 \mathrm{GHz}$. In addition the amplifier has to be compatible with the magnetic field and operating temperatures.

Magnetic trap design: To increase the observation time of an electron, electrons are trapped by magnetic fields. The magnetic field influences the particle trajectory and averaged magnetic field seen by the electron which in turn influences the frequency spectrum of the detectable signal. The magnetic trap designs have to be done with several side constraints based on the detectability of the signal.

Magnetic field homogeneity: Even with a good calibration of the magnetic field, uncertainty in (radial) position reconstruction would lead to huge uncertainty if gradients are present. Thus the magnetic field shape needs to be precisely known and as homogeneous as possible except the gradient needed to trap the electrons. Shimming of the external field and characterization is a key point.

Simulation of CRES: To understand the signal shape and all interdependencies a comprehensive motion and radiation simulation needs to be developed. Project 8 has its own CRES simulation package including antenna response support and digitization electronics simulation called LOCUST [10] which uses the KASSIOPEIA software [11] for particle tracking.

Triggering and reconstruction: Due to the large number of independent antennas used to detect signals in free space and bandwidth needed, initial data rates are high. As the radial electron position in the detection volume is a priori unknown, the individual signals have to be summed using position dependent phase delays before triggering. The data rates require processing, reconstruction and triggering in real time. Advanced techniques like Matched Filtering and Deep Learning are 
investigated. In addition artificial signal sources in antenna arrays are setup in the lab to test triggering and reconstruction ideas.

Calibration concepts: To transfer the high precision frequency spectrum into a high precision energy spectrum, the magnetic field has to be precisely calibrated. An electron gun is under construction which accelerates electrons by electric fields, to well defined electron energies. Using these electrons from a narrow energy band and position, the instrumental response can be calibrated and the central magnetic field in the detection volume can be validated.

\subsection{Atomic Tritium Demonstrator}

The aim of the Atomic Tritium Demonstrator is to develop a cold atomic tritium beam $(<50 \mathrm{mK}$ temperatures) suitable for trapping of neutral atoms. This demonstrator is first developed using hydrogen and deuterium. The production of cold, trapped atomic tritium involves several steps:

Cracking: Starting with molecular tritium, the tritium atoms are dissociated into atomic hydrogen $\left(\mathrm{H}_{2} \rightarrow 2 \mathrm{H}\right)$ using a heated capillary. For sufficient atomic tritium densities required, the cracking rate has to be high. Several test stands are under construction and cracking at high-flows in lab has been demonstrated.

Accommodation: The atomic beam from the cracker is hot $(\sim 2500 \mathrm{~K})$ and has to be cooled down to temperatures of $O(10 \mathrm{~K})$. Development of an accommodator is the next step.

Velocity and state selection: Using a quadrupole magnetic guide, low-field seeking atoms from the accommodator are confined and cooled during transport to the atomic trap.

Field step: A $\sim 1 \mathrm{~T}$ field step is used to decelerate the atoms before going into the atom trap.

Trapping: A neutral atom trap needs to be designed. Two scenarios under consideration are using a Ioffe trap or a trap based on Halbach arrays and concept studies are underway.

\section{Conclusions}

The CRES technique is a promising method to measure neutrino masses using the highprecision spectroscopy near the tritium endpoint. However several R\&D efforts are needed and on going to scale the technique and make it compatible with atomic tritium.

\section{References}

[1] L. I. Bodine, D. S. Parno, and R. G. H. Robertson, Physical Review C 91, 035505 (2015).

[2] E. Fermi, Zeitschrift für Physik 88, 161 (1934).

[3] M. Aker et al., "First direct neutrino-mass measurement with sub-eV sensitivity," (2021), arXiv:2105.08533.

[4] B. Monreal and J. A. Formaggio, Physical Review D 80, 051301 (2009).

[5] Project 8 Collaboration, D. M. Asner, et al., Physical Review Letters 114, 162501 (2015).

[6] A. A. Esfahani et al., Journal of Physics G: Nuclear and Particle Physics 44, 054004 (2017).

[7] Project 8 Collaboration, A. A. Esfahani, et al., Physical Review C 99, 055501 (2019).

[8] C. Claessens for Project 8 Collaboration, "First tritium endpoint measurement with Cyclotron Radiation Emission Spectroscopy (CRES),” (2021), TAUP 2021.

[9] C. Macklin et al., Science 350, 307 (2015).

[10] Project 8 Collaboration, A. A. Esfahani, et al., New Journal of Physics 21, 113051 (2019).

[11] D. Furse et al., New Journal of Physics 19, 053012 (2017). 